Received: $\quad 2019.02 .11$

Accepted: 2019.04.02

Published: 2019.07 .27

\title{
Development and Validation of a Nomogram for Predicting Survival in Patients with Thyroid Cancer
}

Authors' Contribution: Study Design A Data Collection B Statistical Analysis C Data Interpretation D Manuscript Preparation E Literature Search F Funds Collection G

\author{
AE 1,2 Qian Wen* \\ ADE 3,4 Yong Yu* \\ C 1 Jin Yang \\ C 5 Xinwen Wang \\ B 2 Jian Wen \\ B 6 Yuting Wen \\ B 6 Yi Wang \\ A 1 Jun Lyu
}

\author{
1 Clinical Research Center, The First Affiliated Hospital of Xi'an Jiaotong University, \\ Xi'an, Shaanxi, P.R. China \\ 2 Physical Examination Center, The Ninth Hospital of Xi'an Affiliated Hospital of \\ Xi'an Jiaotong University, Xi'an, Shaanxi, P.R. China \\ 3 Department of Oncology, The Second Affiliated Hospital of Xi'an Jiaotong \\ University, X'an, Shaanxi, P.R. China \\ 4 Department of Hepatobiliary and Thoracic Surgery, Shaanxi Provincial Corps \\ Hospital of the Chinese People's Armed Police Force, Xi'an, Shaanxi, P.R. China \\ 5 Department of Foot and Ankle Surgery, Honghui Hospital, Xi'an Jiaotong \\ University, Xi'an, Shaanxi, P.R. China \\ 6 Department of Pathology, The Ninth Hospital of Xi'an Affiliated Hospital of Xi'an \\ Jiaotong University, Xi'an, Shaanxi, P.R. China
}

Corresponding Author: Source of support:
* Qian Wen and Yong Yu have contributed equally to this work Jun Lyu, e-mail: lujun2006@xjtu.edu.cn Departmental sources

Background: The AJCC staging system is inadequate for use in patients with thyroid carcinomas. Here, we aimed to establish a nomogram for thyroid cancer, and we compare its prognostic value with the AJCC staging system in adults diagnosed with thyroid carcinoma.

Material/Methods: Patient records were obtained from the Surveillance, Epidemiology, and End Result database. The 8491 included patients were divided into a modeling cohort $(n=5943)$ and a validation cohort $(n=2548)$. The variables included in the modeling cohort were selected using a backward stepwise selection method with Cox regression, and the prognosis nomogram was constructed. In the validation cohort, we compared our survival model with the AJCC prognosis model using the concordance index, the area under the time-dependent receiver operating characteristic curve, the net reclassification improvement, the integrated discrimination improvement, calibration plotting, and decision curve analysis.

Results: Twelve independent prognostic factors were identified and used to establish the nomogram. In particular, marital status was included in a survival prediction model of thyroid cancer for the first time. The concordance index, area under the time-dependent receiver operating characteristic curve, net reclassification improvement, integrated discrimination improvement, calibration plotting, and decision curve analysis for the nomogram showed better performance compared to the AJCC staging system.

Conclusions: We have developed and validated a highly accurate thyroid cancer prognosis nomogram. The prognostic value of the nomogram is better than that of the AJCC staging system alone.

MeSH Keywords:

Abbreviations:

Full-text PDF:

\section{Disease-Free Survival • Nomograms • Thyroid Neoplasms}

SEER - Surveillance, Epidemiology, and End Result; C-index - concordance index; AUC - area under the time-dependent receiver operating characteristic curve (); NRI - net reclassification improvement; IDI - integrated discrimination improvement; DCA - decision curve analysis; PTC - papillary thyroid carcinoma; FTC - follicular thyroid carcinoma; ATC - anaplastic thyroid carcinoma; MTC - medullary thyroid carcinoma; DTC - differentiated thyroid cancer

https://www.medscimonit.com/abstract/index/idArt/915620

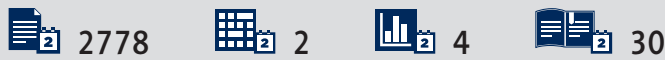




\section{Background}

There were approximately 567000 new cases of thyroid cancer worldwide in 2018, and the global incidence rate is 3 times higher in women (10.2 per 100000$)$ than in men. The mortality rate of thyroid cancer ranges from 0.4 to 0.5 per 100000 in both men and women, with an estimated 41000 deaths annually. In the USA, thyroid cancer incidence rates were about 6.9 per 100000 in men and 23.1 per 100000 in women in 2018. Incidence rates are 4 and 5 times higher in men and women in the United States than in countries with lower Human Development Index. The incidence is increasing faster than that for any other solid tumor, ranking in ninth place for incidence in 2018 [1], and it will replace colorectal cancer as the fourth leading cancer diagnosis by 2030 in the USA [2].

Papillary thyroid carcinoma (PTC), follicular thyroid carcinoma (FTC), anaplastic thyroid carcinoma (ATC), and medullary thyroid carcinoma (MTC) arising from thyroid parafollicular cells are the main pathological types of thyroid carcinoma [3]. Differentiated thyroid cancer (DTC) includes papillary and follicular cancer. PTC constitutes $90 \%$ of thyroid cancers, and they are a major differentiated adenocarcinoma that shows papillary proliferation pathologically. Most cases have an excellent prognosis, but approximately $10 \%$ of PTC patients exhibit recurrences such as lymph node recurrence and lung metastasis [4], or even death. Clinicopathologically, age $>45$ years, extra-thyroidal invasion, distant metastasis, large tumor, vascular invasion, and poorly-differentiated histology are well known factors predicting poor prognois [5]. Most FTCs are minimally invasive, with only slight tumor capsular invasion, and rarely cause distant metastasis [6]. Although much less common, FTC is widely in vasive, with $80 \%$ of these tumors causing distant metastasis, causing a high mortality rate of around $20 \%$ [5]. The poor prognostic factors are large tumor, distant metastasis, extra-thyroidal extension, extensive vascular invasion, age $>45$ years, and wide invasion [7]. ATC is an extremely aggressive undifferentiated tumor, with almost $100 \%$ disease-specific mortality [8], and while representing about only $<2 \%$ of thyroid cancers, they are responsible for $40 \%$ of thyroid cancer deaths. The median survival time from diagnosis is around 6 months [9]. ATC extensively invades into surrounding structures, and distant metastases are observed at the diagnosis in one-third of ATC patients. Most (>70\%) of the patients are women, and the peak age of patients is older than that of DTC [10]. MTC represents $<5 \%$ of thyroid carcinomas; that is, neuroendocrine tumors originating from parafollicular cells of the ultimobranchial body of the neural crest, and it secrets calcitonin. Most (70-80\%) MTCs are sporadic, with $20-30 \%$ being familial [11]. The peak age is younger for familial MTC (approximately 35 years) than for sporadic MTC (40-60 years). The overall 5-year survival rate of patients with MTC is $86 \%$. Poor prognostic factors include older age at diagnosis, the presence of lymph node metastasis at diagnosis, advanced stage, and somatic RET mutation [12]. Adequate risk stratification is crucial in malignant neoplastic disease to avoid both the undertreatment of high-risk subjects and the overtreatment of low-risk patients [13]. For thyroid cancer, surgery and radioiodine therapy remain the established therapeutic procedures. However, the effect of chemotherapy is not clear [14]. This means that personalized treatment according to the potential prognosis for individuals with thyroid cancer is critically important.

Suh et al. demonstrated that the AJCC staging system predicted patient outcome more accurately than other staging systems, which suggests that it is a reliable and cost-effective predictor of outcome in patients with PTC [15]. However, the current AJCC staging system for MTC appears to be less than optimal in distinguishing the risk of mortality among different stage groups [16]. The AJCC staging system can predict the outcome in patients with PTC, but it is inadequate in patients with FTC, MTC, and ATC. This is because factors other than the AJCC stage also influence the prognosis and metastasis of thyroid cancer, including the involvement of lymph nodes [17], age at diagnosis, completion of surgical resection, and pathological subtype [18]. We therefore established a comprehensive prognostic nomogram including all of the factors mentioned above and determined whether its performance is better than that of the AJCC staging system in patients diagnosed with PTC, FTC, ATC, and MTC.

\section{Material and Methods}

\section{Patients}

We reviewed patient data from the latest version of the SEER (Surveillance, Epidemiology, and End Result) database using SEER*Stat version 8.3.5 released on March 6, 2018 (https:// seer.cancer.gov/). We searched for patients whose ICD-0-3/ WHO 2008 histological type codes were 8330/3 (FTC), 8050/3 (PTC), 8510/3 (MTC), or 8021/3 (ATC). We also searched for patients with a positive diagnostic confirmation in histology, categorized as either alive or with thyroid carcinoma as the cause of death, and as active follow-up. We excluded patients under the age of 18 years, those initially confirmed by a death certificate or only an autopsy, and cases with unknown or incomplete important variables.

There were 8491 patients identified from the SEER database between 2004 and 2015. These patients were randomly divided into a modeling cohort $(n=5943,70 \%)$ for constructing the prognosis nomogram and a validation cohort $(n=2548$, $30 \%)$ for evaluating the constructed nomogram. This retrospective study was exempted from consent requirements by the SEER database administrators. 
Table 1. Clinical and pathological characteristics of patients in the modeling and validation cohorts.

\begin{tabular}{|c|c|c|c|c|c|}
\hline \multirow[b]{2}{*}{ Number of Patients n (\%) } & \multicolumn{2}{|c|}{ Modeling cohort } & \multicolumn{2}{|c|}{ Validation cohort } & \multirow[t]{2}{*}{ P-value } \\
\hline & 5943 & (70) & 2548 & $(30)$ & \\
\hline Median age at diagnosis, year (interquartile range) & 50 & $(39-62)$ & 50 & $(39-60)$ & 0.16 \\
\hline Sex n (\%) & & & & & 0.84 \\
\hline Male & 1680 & $(28.3)$ & 714 & $(28.0)$ & \\
\hline Female & 4263 & $(71.7)$ & 1834 & $(72)$ & \\
\hline Race $\mathrm{n}(\%)$ & & & & & 0.89 \\
\hline White & 4883 & $(82.2)$ & 2094 & $(82.2)$ & \\
\hline Black & 556 & $(9.4)$ & 232 & $(9.1)$ & \\
\hline Other & 504 & $(8.5)$ & 222 & $(8.7)$ & \\
\hline Marital status n (\%) & & & & & 0.93 \\
\hline Married & 3691 & $(62.1)$ & 1573 & $(61.7)$ & \\
\hline Unmarried & 1983 & (33.4) & 861 & (33.8) & \\
\hline Unknown & 269 & $(4.5)$ & 114 & $(4.5)$ & \\
\hline Insurance recode $\mathrm{n}(\%)$ & & & & & 0.89 \\
\hline Uninsured & 142 & $(2.4)$ & 65 & $(2.6)$ & \\
\hline Insured and any medical & 4234 & (71.2) & 1807 & (70.9) & \\
\hline Unknown & 1567 & $(26.4)$ & 676 & $(26.5)$ & \\
\hline Tumor size $n(\%)$ & & & & & 0.06 \\
\hline$\leq 50 \mathrm{~mm}$ & 5016 & $(84.4)$ & 2190 & $(85.9)$ & \\
\hline $50-100 \mathrm{~mm}$ & 748 & $(12.6)$ & 285 & $(11.2)$ & \\
\hline$>100 \mathrm{~mm}$ & 63 & $(1.1)$ & 16 & $(0.6)$ & \\
\hline No/unknown & 116 & $(2.0)$ & 57 & $(2.2)$ & \\
\hline AJCC n (\%) & & & & & 0.28 \\
\hline 1 & 3296 & $(55.5)$ & 1470 & $(57.7)$ & \\
\hline II & 856 & $(14.4)$ & 344 & $(13.5)$ & \\
\hline III & 821 & $(13.8)$ & 343 & $(13.5)$ & \\
\hline IV & 970 & $(16.3)$ & 391 & $(15.3)$ & \\
\hline Derived AJCC T n (\%) & & & & & 0.06 \\
\hline TO & 15 & $(0.3)$ & 8 & $(0.3)$ & \\
\hline $\mathrm{T} 1$ & 2434 & $(41.0)$ & 1116 & $(43.8)$ & \\
\hline $\mathrm{T} 2$ & 1492 & $(25.1)$ & 623 & $(24.5)$ & \\
\hline $\mathrm{T} 3$ & 1393 & $(23.4)$ & 533 & $(20.9)$ & \\
\hline $\mathrm{T} 4$ & 609 & $(10.2)$ & 268 & $(10.5)$ & \\
\hline Derived AJCC N n (\%) & & & & & 0.83 \\
\hline No & 4739 & (79.7) & 2026 & (79.5) & \\
\hline N1 & 1204 & $(20.3)$ & 522 & $(20.5)$ & \\
\hline
\end{tabular}


Table 1 continued. Clinical and pathological characteristics of patients in the modeling and validation cohorts.

\begin{tabular}{|c|c|c|c|c|c|}
\hline & \multicolumn{2}{|c|}{ Modeling cohort } & \multicolumn{2}{|c|}{ Validation cohort } & $\begin{array}{c}\text { P-value } \\
0.11\end{array}$ \\
\hline MO & 5540 & $(93.2)$ & 2400 & $(94.2)$ & \\
\hline M1 & 403 & (6.8) & 148 & (5.8) & \\
\hline Extent of disease $n(\%)$ & & & & & 0.07 \\
\hline Localized & 3268 & $(55.0)$ & 1443 & $(56.6)$ & \\
\hline Regional & 2129 & $(35.8)$ & 909 & $(35.7)$ & \\
\hline Distant & 546 & $(9.2)$ & 196 & (7.7) & \\
\hline ICD-O-3 histology n (\%) & & & & & 0.44 \\
\hline Papillary carcinoma & 2349 & $(39.5)$ & 1050 & $(41.2)$ & \\
\hline Follicular carcinoma & 2284 & $(38.4)$ & 942 & $(37)$ & \\
\hline Medullary carcinoma & 959 & $(16.1)$ & 415 & $(16.3)$ & \\
\hline Anaplastic carcinoma & 351 & (5.9) & 141 & (5.5) & \\
\hline Surgery n (\%) & & & & & 0.54 \\
\hline Yes & 5703 & $(96.0)$ & 2453 & $(96.3)$ & \\
\hline No & 240 & $(4.0)$ & 95 & $(3.7)$ & \\
\hline Radiation n (\%) & & & & & 0.85 \\
\hline Yes & 2660 & $(44.8)$ & 1134 & $(44.5)$ & \\
\hline None/unknown & 3283 & $(55.2)$ & 1414 & $(55.5)$ & \\
\hline Chemotherapy n (\%) & & & & & 0.77 \\
\hline Yes & 236 & $(4.0)$ & 97 & (3.8) & \\
\hline No & 5707 & (96) & 2451 & $(96.2)$ & \\
\hline Median follow-up, months (interquartile range) & 66 & $(26-104)$ & 66 & $(26-104)$ & 0.78 \\
\hline Thyroid cancer-specific mortality (\%) & 495 & $(8.3)$ & 191 & $(7.5)$ & 0.21 \\
\hline
\end{tabular}

Race-Other - American Indian \& AK Native \& Asian \& Pacific Islander. Marital status - Unmarried: Single \& Separated \& Divorced \& Widowed \& Unmarried or Domestic Partner.

Variables from the SEER database were selected by a backward stepwise selection method in the Cox regression model. The following variables were examined: age at diagnosis, sex, race, insurance recode, marital status, tumor size, AJCC stage, derived AJCC stage T, derived AJCC stage $N$, derived AJCC stage $M$, extent of disease, ICD-0-3 histology, surgery, radiation, and chemotherapy. All variables except sex, race, and insurance recode were entered into the nomogram. Death was attributed to thyroid cancer if this was listed as the cause of death on the death certificate.

\section{Statistical analyses}

All statistical analyses were performed using SPSS (version 21.0) and $R$ software. A 2-sided probability value of $P \leq 0.05$ was considered to be statistically significant. Mean \pm SD values were used to express continuous variables conforming to a normal distribution, and all other variables were expressed as median $\left(25^{\text {th }}-75^{\text {th }}\right.$ percentile) values. Variables were included in the multivariable Cox regression analyses at $\mathrm{P} \leq 0.1$.

Statistical analyses to identify risk factors were performed by applying the backward stepwise selection method of Cox regression to the modeling cohort. All related statistical analyses and the establishment of the nomogram were performed using R software. The discrimination performance of our nomogram was compared with that of the traditional AJCC staging system by measuring the concordance index ( $\mathrm{C}$-index) and the area under the time-dependent receiver operating characteristic curve (AUC). In addition 
Table 2. Selected variables by multivariate Cox regression analysis (modeling cohort).

\begin{tabular}{|c|c|c|c|}
\hline \multirow{2}{*}{ Variables } & \multicolumn{3}{|c|}{ Multivariate analysis } \\
\hline & HR & $95 \% \mathrm{CI}$ & P-value \\
\hline Age at diagnosis & 1.03 & $1.03-1.04$ & $<0.01$ \\
\hline \multicolumn{4}{|l|}{ Marital status n (\%) } \\
\hline Married & & Reference & \\
\hline Unmarried & 1.47 & $1.22-1.77$ & $<0.01$ \\
\hline Unknown & 0.32 & $0.15-0.67$ & $<0.01$ \\
\hline \multicolumn{4}{|l|}{ Tumor size $\mathrm{n}(\%)$} \\
\hline$\leq 50 \mathrm{~mm}$ & & Reference & \\
\hline $50-100 \mathrm{~mm}$ & 1.32 & $1.05-1.65$ & 0.02 \\
\hline$>100 \mathrm{~mm}$ & 1.93 & $1.30-2.85$ & $<0.01$ \\
\hline NO/unknown & 1.69 & $1.24-2.31$ & $<0.01$ \\
\hline \multicolumn{4}{|l|}{ AJCC n (\%) } \\
\hline 1 & & Reference & \\
\hline ॥ & 2.86 & $1.13-7.26$ & 0.03 \\
\hline III & 2.04 & $0.88-4.71$ & 0.10 \\
\hline IV & 11.32 & $5.32-24.07$ & $<0.01$ \\
\hline \multicolumn{4}{|l|}{ Derived AJCC T n (\%) } \\
\hline TO & & Reference & \\
\hline $\mathrm{T} 1$ & 2.15 & $0.60-7.69$ & 0.24 \\
\hline $\mathrm{T} 2$ & 1.87 & $0.52-6.67$ & 0.34 \\
\hline T3 & 3.54 & $1.08-11.60$ & 0.04 \\
\hline $\mathrm{T} 4$ & 7.9 & $2.46-25.70$ & $<0.01$ \\
\hline \multicolumn{4}{|l|}{ Derived AJCC N n (\%) } \\
\hline NO & & Reference & \\
\hline N1 & 1.21 & $0.98-1.49$ & 0.08 \\
\hline \multicolumn{4}{|l|}{ Derived AJCC M n (\%) } \\
\hline MO & & Reference & \\
\hline M1 & 2.18 & $1.60-2.95$ & $<0.01$ \\
\hline \multicolumn{4}{|l|}{ Extent of disease $n(\%)$} \\
\hline Localized & & Reference & \\
\hline Regional & 1.502 & $0.9603-2.3479$ & 0.074 \\
\hline Distant & 1.863 & $1.1019-3.1512$ & 0.02 \\
\hline \multicolumn{4}{|l|}{ ICD-O-3 histology n (\%) } \\
\hline Papillary carcinoma & & Reference & \\
\hline Follicular carcinoma & 1.74 & $1.17-2.60$ & 0.01 \\
\hline Medullary carcinoma & 1.59 & $1.06-2.40$ & 0.03 \\
\hline Anaplastic carcinoma & 6.32 & $4.22-9.45$ & $<0.01$ \\
\hline
\end{tabular}


Table 2 continued. Selected variables by multivariate Cox regression analysis (modeling cohort).

\begin{tabular}{|c|c|c|c|}
\hline \multirow{2}{*}{ Variables } & \multicolumn{3}{|c|}{ Multivariate analysis } \\
\hline & HR & $95 \% \mathrm{Cl}$ & P-value \\
\hline \multicolumn{4}{|l|}{ Surgery n (\%) } \\
\hline Yes & & Reference & \\
\hline No & 2.26 & $1.79-2.85$ & $<0.01$ \\
\hline \multicolumn{4}{|l|}{ Radiation n (\%) } \\
\hline Yes & & Reference & \\
\hline None/unknown & 1.39 & $1.14-1.68$ & $<0.01$ \\
\hline \multicolumn{4}{|l|}{ Chemotherapy n (\%) } \\
\hline Yes & & Reference & \\
\hline No & 1.25 & $0.99-1.58$ & 0.06 \\
\hline
\end{tabular}

Marital status - Unmarried: Single \& Separated \& Divorced \& Widowed \& Unmarried or Domestic Partner.

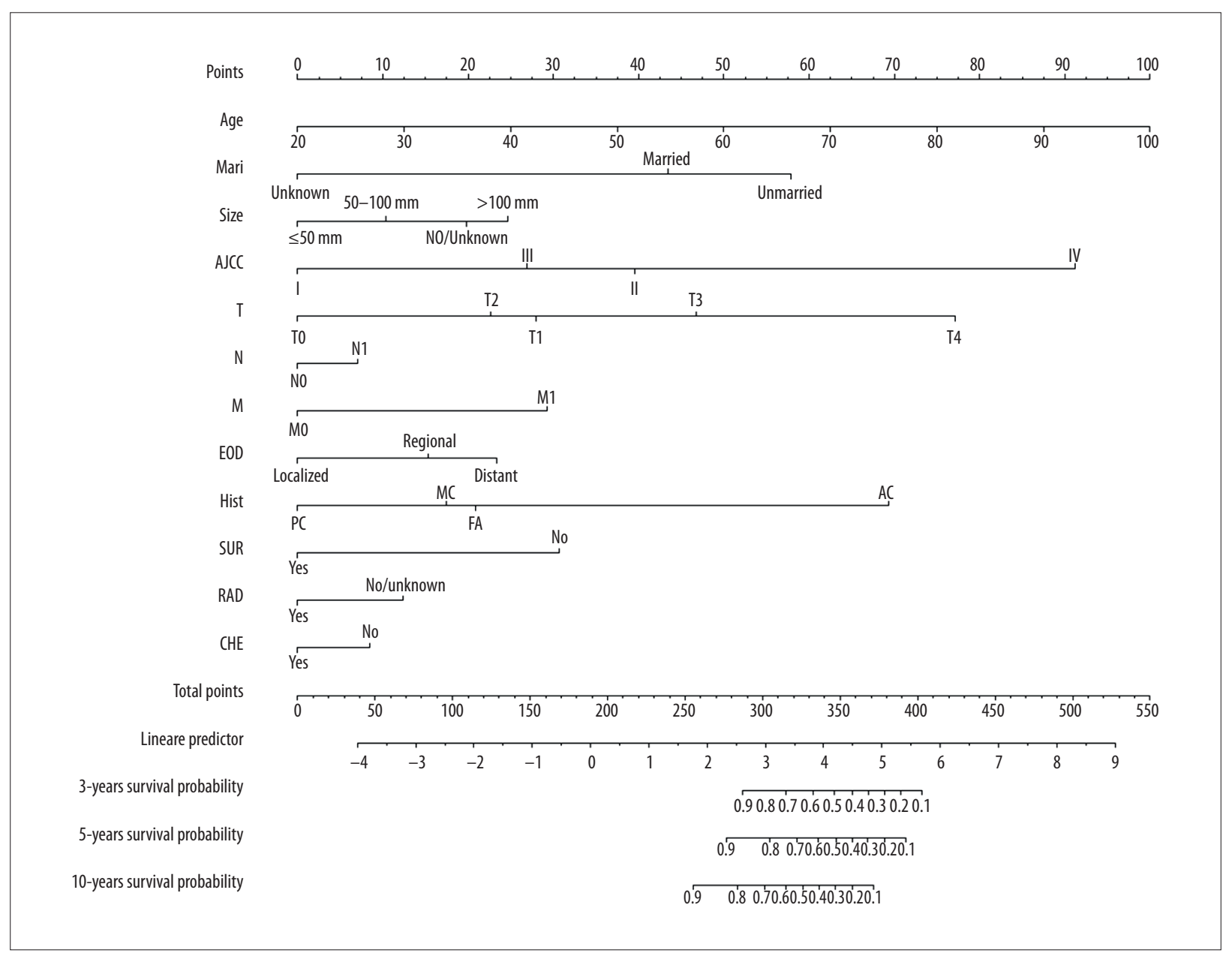

Figure 1. Nomogram predicting 3-year, 5-year, and 10-year survival. Unmarried: Single \& Separated \& Divorced \& Widowed \& Unmarried or Domestic Partner. EOD - extent of disease. SUR - surgery; RAD - radiation; CHE - chemotherapy. Hist - ICD-O-3 histology. PC - papillary carcinoma; FA - follicular carcinoma; MC - medullary carcinoma; AC - anaplastic carcinoma. 


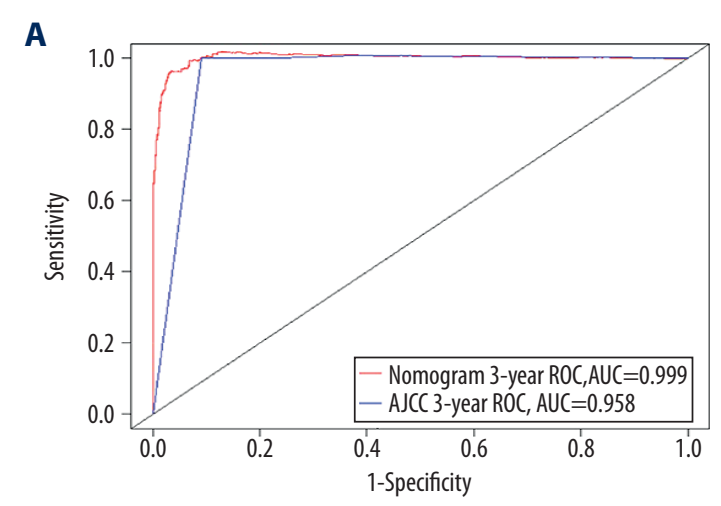

B

C
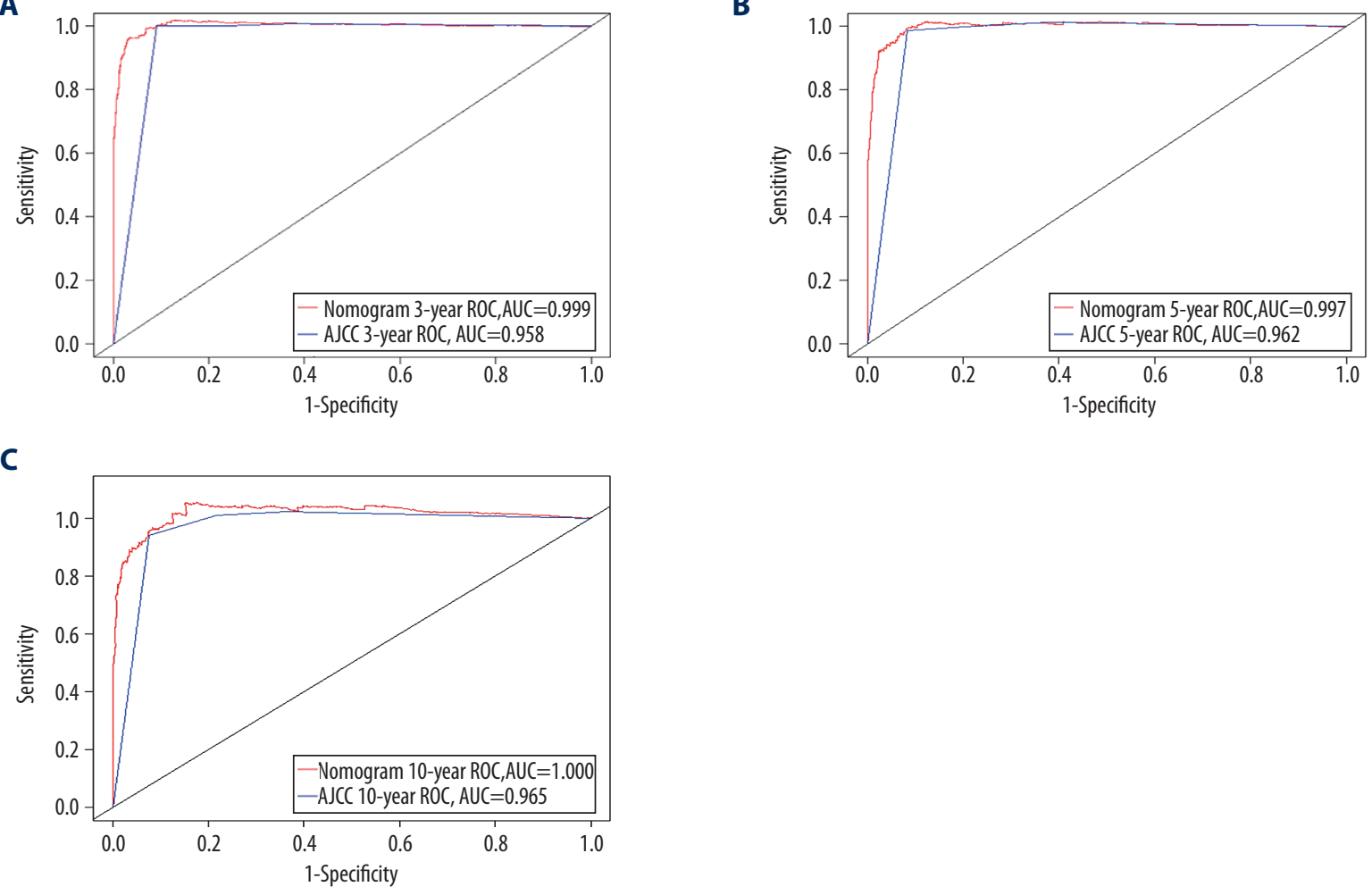

Figure 2. ROC curves. The ability of the model to be measured by the C-index. In the validation cohort, predicted probabilities for 3-,5and 10-years survival (A-C) based on the nomogram and AJCC in the validation sets.

to these indices, we calculated the integrated discrimination improvement (IDI) and the net reclassification improvement (NRI) to evaluate the predictive accuracy of our model. Calibration plots were generated to evaluate the predictive performance by comparing the nomogram-predicted and actual observed 3-, 5-, and 10-year survival rates. Decision curve analysis (DCA) was used to evaluate the clinical usefulness of the nomogram by quantifying the net benefits at different threshold probabilities.

\section{Results}

\section{Clinicopathological characteristics of the patients}

The median age at the time of diagnosis was 50 years in both the modeling and validation cohorts. Most of the patients in both cohorts were female, white, and married, had insurance and any medical and a tumor size of $\leq 5 \mathrm{~mm}$, and were in AJCC stage I, derived AJCCT1, derived AJCC stage N0, and derived AJCC stage MO. Most of the patients had a localized tumor and a PTC or FTC in both cohorts, and received surgery but not radiation or chemotherapy. The median follow-up time was 66 months in both cohorts. The demographics and tumor characteristics of the patients are summarized in Table 1.

\section{Independent prognostic factors in the modeling cohort}

The variables of age at diagnosis, marital status, tumor size, AJCC stage, derived AJCC stage T, derived AJCC stage $N$, derived AJCC stage $M$, extent of disease, histology, surgery, radiation, and chemotherapy were entered into the multivariable Cox regression analyses. The multivariate analyses revealed the following significant risk factors for survival: age at diagnosis (hazard ratio $[H R]=1.034, P<0.001$ ), being unmarried $(\mathrm{HR}=1.468, \mathrm{P}<0.001$ vs. married), AJCC stage II $(\mathrm{HR}=2.863$, $\mathrm{P}=0.027$ vs. AJCC stage I), AJCC stage IV $(H R=11.317, \mathrm{P}<0.001$ vs. AJCC stage I), derived AJCC stage T3 $(\mathrm{HR}=3.535 \mathrm{P}=0.037$ vs. derived AJCC stage T0), derived AJCC stage T4 (HR=7.944, $\mathrm{P}<0.001$ vs. derived AJCC stage T0), derived AJCC stage M1 $(H R=2.175, P<0.001$ vs. derived AJCC stage M0), distant extent of disease $(H R=1.863 P=0.02$ vs. localized extent of disease), follicular histology ( $\mathrm{HR}=1.744, \mathrm{P}=0.007$ vs. papillary histology), medullary histology $(\mathrm{HR}=1.593, \mathrm{p}=0.026 \mathrm{vs}$. papillary histology), anaplastic histology $(H R=6.316, P<0.001$ vs. papillary histology), no surgery ( $\mathrm{HR}=2.261, \mathrm{P}<0.001$ vs surgery), and no/unknown radiation ( $\mathrm{HR}=1.388, \mathrm{P}<0.001$ vs. radiation). In particular, we found that tumor size was also a risk factor affecting survival: $H R=1.316(P=0.017)$ for $50-100 \mathrm{~mm}$ vs. $\leq 50 \mathrm{~mm}$, and $H R=1.925$ ( $P=0.001)$ for $>100 \mathrm{~mm}$ vs. $\leq 50 \mathrm{~mm}$ (Table 2$)$. 

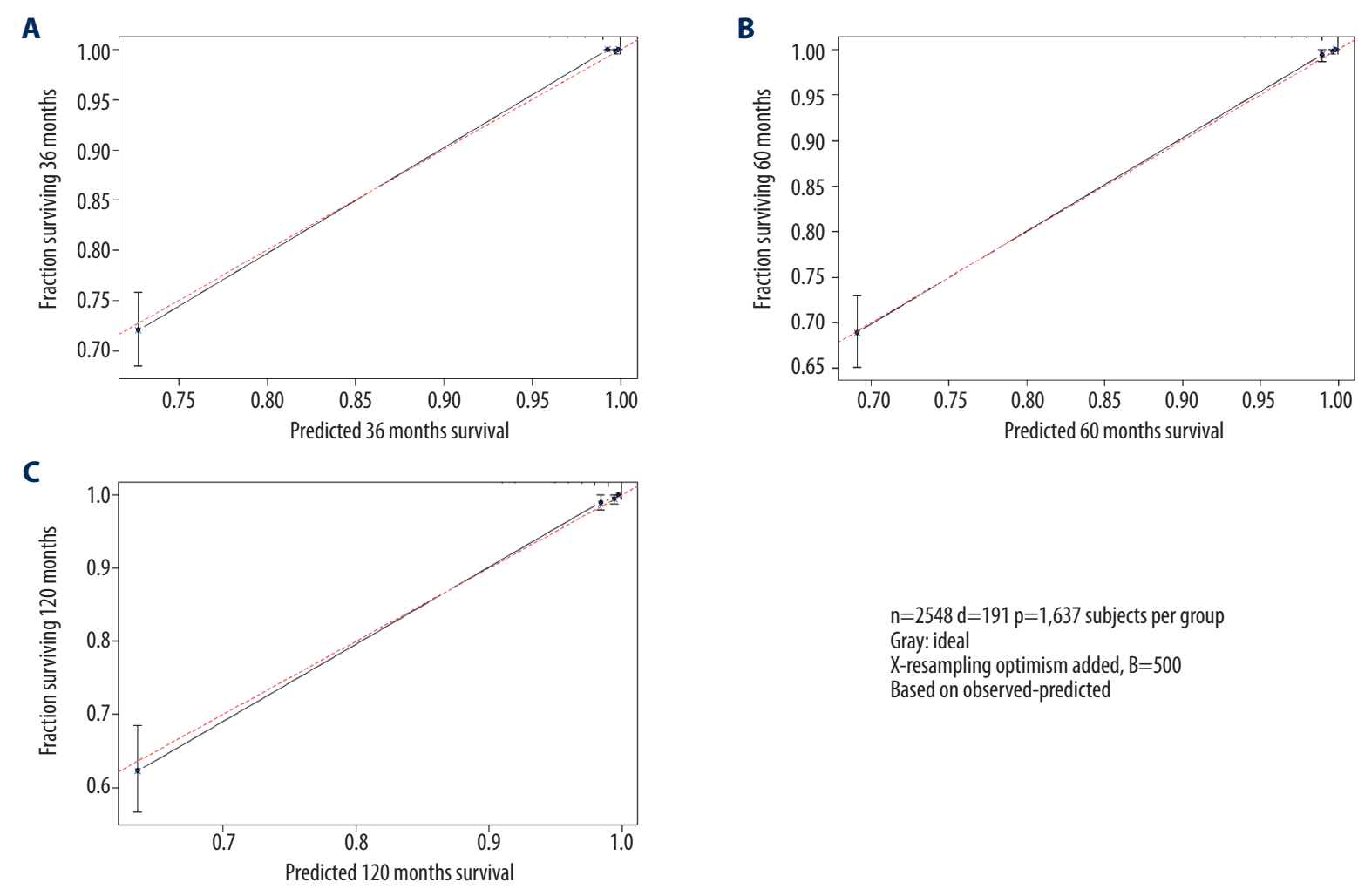

$n=2548 d=191 p=1,637$ subjects per group Gray: ideal

$X$-resampling optimism added, $B=500$

Based on observed-predicted

Figure 3. Calibration plots. These show the relationship between the predicted probabilities for 3-, 5- and 10-years survival (A-C) based on the nomogram and actual values in the validation sets.

\section{Prognostic nomogram for 3-, 5-, and 10-year survival rates}

Based on the significant independent factors that were selected variables with HRs, we constructed a nomogram for predicting the 3-, 5-, and 10-year survival rates in the modeling cohort. The nomogram showed that the age at diagnosis was the strongest contributor to the prognosis, followed by the AJCC stage, derived AJCC stage T, histology, marital status, surgery, derived AJCC stage $M$, tumor size, extent of disease, radiation, chemotherapy, and derived AJCC stage N. Each variable was given a score on a scale. These scores were then added to obtain the total score, and a vertical line was dropped down from the total points row to estimate the 3-, 5-, and 10 year survival rates (Figure 1).

\section{Validation of the prognostic nomogram}

The prognostic nomogram and the AJCC staging system were compared using the verification cohort. The C-index was higher for the nomogram than for the AJCC staging system ( 0.975 vs. 0.929), as were the AUCs for the nomogram $(0.999,0.997$, and 1.000 for 3-, 5-, and 10-year survival rates, respectively, vs. $0.958,0.962$, and 0.965 , respectively), which indicated the good discriminative ability of the nomogram (Figure 2). Compared with the AJCC stage, the NRIs for 3, 5, and 10 years of followup were 0.643 (95\% confidence interval $[\mathrm{Cl}]=0.515-0.739)$, $0.565(95 \% \mathrm{Cl}=0.452-0.674)$, and $0.549(95 \% \mathrm{Cl}=0.423-0.767)$, respectively; the corresponding IDIs were $0.264,0.271$, and 0.241 , respectively (all $\mathrm{P}<0.001$ ). These indicators demonstrate that the nomogram showed better discrimination performance than the AJCC staging system.

Calibration plots of the nomogram showed that the predicted 3-, 5-, and 10-year survival rates for the modeling and validation groups were almost identical to the actual observations (Figure 3).

These results show that although both models yield net benefits, the 3-, 5-, and 10-year DCA curves for the nomogram yielded net benefits greater than when using the traditional AJCC staging system in the validation set (Figure 4).

\section{Discussion}

The incidence of thyroid cancer is reportedly 3- to 4-fold higher among females than males worldwide, ranking as the sixth most common malignancy diagnosed in women [3]. Thyroid 

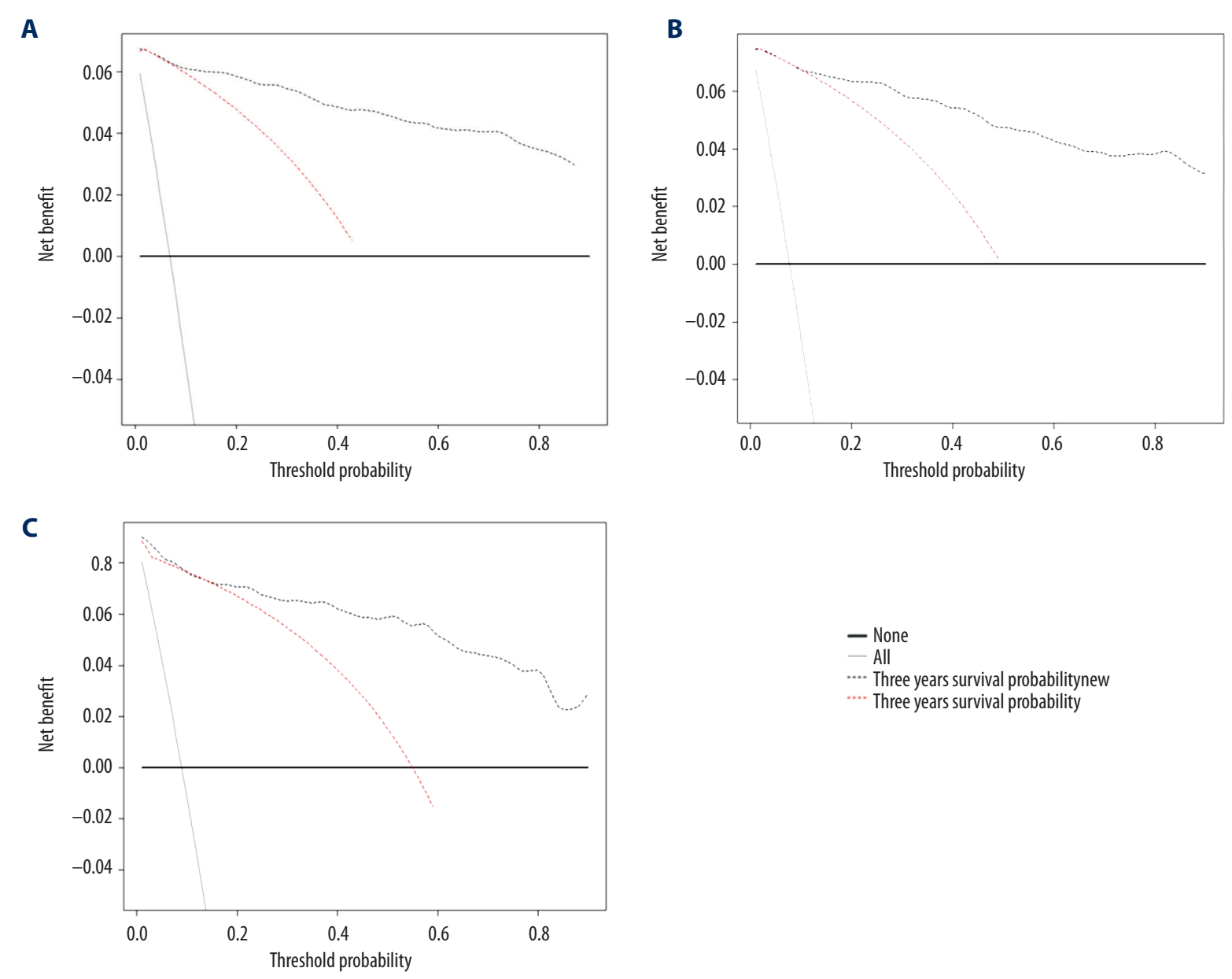

Figure 4. Decision curve analysis. In the figure, the abscissa is the threshold probability and the ordinate is the net benefit rate. The horizontal one indicates that all samples are negative and all are not treated, with a net benefit of zero. The oblique one indicates that all samples are positive. The net benefit is a backslash with a negative slope. A-C show prediction for 3-, 5and 10-year survival in the validation sets. Survival probability new: the nomogram. Survival probability: AJCC.

cancer can occur at any age, but it is rare in childhood, with most tumors being diagnosed during the third to sixth decades of life [19]. The main pathological types of carcinomas are PTC, FTC, and ATC. MTC arises from thyroid parafollicular cells. PTC constitutes $85-90 \%$ of all thyroid cancer cases, followed by FTC (5-10\%) and MTC (about 2\%). ATC accounts for less than $2 \%$ of thyroid cancers, but it is still an important lethal disease [19]. The 10-year overall relative survival rates for US patients with PTC, FTC, MTC, and undifferentiated carcinoma/ATC are $93 \%, 85 \%, 75 \%$, and $14 \%$, respectively [20].

A nomogram is a useful predictive tool that is tailored to the profile of an individual patient and creates a more precise prediction compared to the traditional AJCC staging system [16]. In recent years, nomograms have been applied in most types of cancer $[3,18]$. However, nomograms have been developed for PTC rather than other types of pathological thyroid carcinoma to generate individualized predictions [21-25], and there has also been an overall lack of evaluations of the developed nomograms. As is well known, characterization according to 2 related properties of discrimination is the most basic assessment for predictive models [26], Moreover, comparisons of calibration methods and the clinical usefulness of predictive models are also very important [27]. We have established a comprehensive prognostic nomogram and compared its prognostic value with the AJCC staging system from the 3 aspects above in patients diagnosed with PTC, FTC, ATC, and MTC.

The multivariate Cox regression performed in this study revealed that age at diagnosis, being unmarried, AJCC stage II, AJCC stage IV, derived AJCC stage T3, derived AJCC stage T4, derived AJCC stage M1, distant extent of disease, follicular, medullary, and anaplastic histology, no surgery, no/unknown radiation, and large tumor are risk factors for survival. It is worth 
noting that we discovered the marital status in a survival prediction model of thyroid cancer for the first time. To the best of our knowledge, there has been no previous description of the effect of marital status on survival of patients with thyroid cancer. This new information can therefore further help clinicians to make more effective clinical decisions.

We have established and validated a nomogram for predicting the survival rates of thyroid cancer patients at 3,5 , and 10 years. Our nomogram model contains risk factors that can be easily collected from historical medical records. The clinical applicability and ease of use are important advantages of the nomogram we constructed. To further determine whether our prognostic model performed better than the traditional AJCC staging system, we evaluated the performance of our survival model using several parameters that are commonly assessed in model validations: C-index, AUC, NRI, IDI, calibration plots, and DCA. The receiver operating characteristic curve and C-statistic are typically used to assess discrimination [28]. The IDI and $\mathrm{NRI}$ were also used in the present study to assess the discrimination performance based on the additional diagnostic value of our model compared to the AJCC staging system. Although the C-index and AUC of the nomogram were only a little higher than those of the AJCC staging system (Figure 2), the addition of the nomogram to the AJCC staging system significantly improved the reclassification performance in the validation cohort: $\mathrm{NRI}=0.643,0.565$, and 0.549 , and $I D I=0.264,0.271$, and 0.241 for 3,5 , and 10 years, respectively (all $P<0.001$ ).

The calibration plots approximating a 45-degree line indicated that the nomogram predictions were well calibrated (Figure 3). DCA is used for evaluating clinical usefulness, and it can show a minimal net benefit of modified scores in an incorporated index. Some studies have demonstrated the benefits of DCA and recommend its use $[29,30]$. The present results indicate that the 3-, 5-, and 10-year DCA curves of our model yielded net benefits greater than those of the traditional AJCC staging system (Figure 4).

Our newly developed nomogram can be used to improve the prediction performance when using the AJCC staging system alone. This supports the use of our nomogram as a tool for helping to redirect and optimize treatment in this clinical setting.

\section{Limitations}

The patients included in this study were mainly white, and so it might not be valid to extrapolate the results to other racial groups. The analyzed data set was extracted from the SEER database, making this a retrospective study with the inevitable inherent bias. Selection bias was present in the selection and exclusion of patients, because we only included those patients with complete information. In addition, many factors were not included, such as RET mutation. The other limitations of this study include the relatively small sample, so more data need to be analyzed to improve the accuracy of model performance assessments. Finally, the values predicted using the nomogram should only be used by clinicians for reference purposes.

\section{Conclusions}

We have developed and validated a highly accurate thyroid cancer prognosis nomogram. The prognostic value of the nomogram is better than that of the AJCC staging system alone. In particular, marital status has been included in a survival prediction model of thyroid cancer for the first time. The nomogram developed in this study may be a valuable tool when explaining 3-, 5-, and 10-year survival rates to patients in clinical practice.

\section{Ethics approval and consent to participate}

The data comes from the SEER database, and due to its retrospective nature, the study was exempted by the SEER database administrators.

\section{Consent for publication}

All patients came from the SEER database (Surveillance, Epidemiology, and End Result), which is publicly available.

\section{Availability of data and material}

The datasets analyzed during current study are available from the corresponding author upon reasonable request.

\section{Acknowledgements}

We thank all colleagues involved in the study for their contributions.

\section{Conflicts of interest}

None. 


\section{References:}

1. Bray F, Ferlay J, Soerjomataram I et al: Global cancer statistics 2018: GLOBOCAN estimates of incidence and mortality worldwide for 36 cancers in 185 countries. Cancer J Clin, 2018; 68: 394-424

2. Rahib L, Smith BD, Aizenberg R et al: Projecting cancer incidence and deaths to 2030: The unexpected burden of thyroid, liver, and pancreas cancers in the United States. Cancer Res, 2014; 74: 2913-21

3. Jemal A, Bray F, Center MM et al: Global cancer statistics. Cancer J Clin, 2011; 61: 69-90

4. Mansour J, Sagiv D, Alon E, Talmi Y: Prognostic value of lymph node ratio in metastatic papillary thyroid carcinoma. J Laryngol Otol, 2018; 132: 8-13

5. Vuong HG, Kondo T, Pham TQ et al: Prognostic significance of diffuse sclerosing variant papillary thyroid carcinoma: A systematic review and metaanalysis. Eur J Endocrinol, 2017; 176: 431-39

6. LiVolsi VA, Asa SL: The demise of follicular carcinoma of the thyroid gland. Thyroid, 1994; 4: 233-36

7. Ito $\mathrm{Y}$, Hirokawa M, Higashiyama T et al: Prognosis and prognostic factors of follicular carcinoma in Japan: importance of postoperative pathological examination. World J Surg, 2007; 31: 1417-24

8. Are C, Shaha AR: Anaplastic thyroid carcinoma: biology, pathogenesis, prognostic factors, and treatment approaches. Ann Surg Oncol, 2006; 13: 453-64

9. Untch BR, Olson JJ: Anaplastic thyroid carcinoma, thyroid lymphoma, and metastasis to thyroid. Surg Oncol Clin N Am, 2006; 15: 661-79

10. Kebebew E, Greenspan FS, Clark $\mathrm{OH}$ et al: Anaplastic thyroid carcinoma. Treatment outcome and prognostic factors. Cancer, 2005; 103: 1330-35

11. Giuffrida D, Gharib H: Current diagnosis and management of medullary thyroid carcinoma. Ann Oncol, 1998; 9: 695-701

12. Elisei $R$, Cosci $B$, Romei $C$ et al: Prognostic significance of somatic RET oncogene mutations in sporadic medullary thyroid cancer: A 10-year followup study. J Clin Endocrinol Metab, 2008; 93: 682-87

13. Krajewska J, Chmielik E, Jarząb B: Dynamic risk stratification in the followup of thyroid cancer: What is still to be discovered in 2017? Endocr Relat Cancer, 2017; 24: R387-402

14. Schmidbauer B, Menhart K, Hellwig D, Grosse J: Differentiated thyroid cancer-treatment: State of the art. Int J Mol Sci, 2017; 18: pii: E1292

15. Suh S, Kim YH, Goh TS et al: Outcome prediction with the revised American joint committee on cancer staging system and American thyroid association guidelines for thyroid cancer. Endocrine, 2017; 58: 495-502

16. Adam MA, Thomas S, Roman SA et al: Rethinking the Current American Joint Committee on Cancer TNM staging system for medullary thyroid cancer. JAMA Surg, 2017; 152: 869-76
17. Shah S, Boucai L: Effect of age on response to therapy and mortality in patients with thyroid cancer at high risk of recurrence. J Clin Endocrinol Metab, 2018; 103(2): 689-97

18. Leboulleux S, Rubino C, Baudin E et al: Prognostic factors for persistent or recurrent disease of papillary thyroid carcinoma with neck lymph node metastases and/or tumor extension beyond the thyroid capsule at initial diagnosis. The Journal of Clin Endocrinol Met, 2005; 90: 5723-29

19. Haddad RI, Lydiatt WM, Ball DW et al: Anaplastic thyroid carcinoma, Version 2.2015. J Natl Compr Canc Netw, 2015; 13: 1140-50

20. Hundahl SA, Fleming ID, Fremgen AM, Menck HR: A National Cancer Data Base report on 53,856 cases of thyroid carcinoma treated in the U.S., 19851995 [see commetns]. Cancer, 1998; 83: 2638-48

21. Wang $Y$, Guan $Q$, Xiang J: Nomogram for predicting central lymph node metastasis in papillary thyroid microcarcinoma: A retrospective cohort study of 8668 patients. INT J Surg, 2018; 55: 98-102

22. Jianyong L, Zhihui L, Rixiang G, Jingqiang Z: Using a nomogram based on preoperative serum fibrinogen levels to predict recurrence of papillary thyroid carcinoma. BMC Cancer, 2018; 18: 390

23. Ge MH, Cao J, Wang JY et al: Nomograms predicting disease-specific regional recurrence and distant recurrence of papillary thyroid carcinoma following partial or total thyroidectomy. Medicine (Baltimore), 2017; 96: e7575

24. Kim SK, Chai YJ, Park I et al: Nomogram for predicting central node metastasis in papillary thyroid carcinoma. J Surg Oncol, 2017; 115: 266-72

25. Hei H, Song Y, Qin J: A nomogram predicting contralateral central neck lymph node metastasis for papillary thyroid carcinoma. J Surg Oncol, 2016; 114: 703-7

26. Alba AC, Agoritsas T, Walsh M et al: Discrimination and calibration of clinical prediction models: Users' guides to the medical literature. JAMA, 2017; 318: 1377-84

27. Walsh CG, Sharman K, Hripcsak G: Beyond discrimination: A comparison of calibration methods and clinical usefulness of predictive models of readmission risk. J Biomed Inform, 2017; 76: 9-18

28. Pencina MJ, D'Agostino RS: Evaluating discrimination of risk prediction models: The C statistic. JAMA, 2015; 314: 1063-64

29. Talluri R, Shete S: Using the weighted area under the net benefit curve for decision curve analysis. BMC Med Inform Decis Mak, 2016; 16: 94

30. Rousson V, Zumbrunn T: Decision curve analysis revisited: overall net benefit, relationships to ROC curve analysis, and application to case-contro studies. BMC Med Inform Decis Mak, 2011; 11: 45 